\title{
Necrose cutânea induzida por antagonistas da vitamina $K$
}

\author{
Skin necrosis induced by vitamin K antagonists \\ Jose Manoel da Silva Silvestre ${ }^{1}$, Fernando Thomazinho ${ }^{2}$, Wander Eduardo Sardinha ${ }^{1}$, \\ Igor Schincariol Perozin ${ }^{3}$, Domingos de Morais Filho ${ }^{1}$
}

\begin{abstract}
Resumo
Os anticoagulantes orais que atuam através do antagonismo à vitamina K são utilizados na prática clínica há muito tempo, porém ainda há dificuldades no seu manejo e na condução das complicações. Entre as complicações, as mais conhecidas são os transtornos hemorrágicos, mas outras também devem ser reconhecidas, tais como a necrose induzida por varfarina. Esta é uma grave, porém rara complicação, cuja fisiopatologia é ainda obscura e cujas causas são indefinidas. Dentre as possíveis causas, as mais prováveis são a deficiência de proteína $\mathrm{C}$ e de proteína $\mathrm{S}$, reações de hipersensibilidade e deficiência de fator VII. Há maior incidência desta complicação entre mulheres de meia-idade, acometendo preferencialmente mamas e glúteos. As medidas mais importantes para o tratamento são: suspensão imediata da droga, uso de heparina não fracionada ou de baixo peso molecular em doses terapêuticas, emprego da vitamina $\mathrm{K}$ e, eventualmente, infusão de plasma fresco congelado ou de proteína $\mathrm{C}$ ativada recombinante.
\end{abstract}

Palavras-chave: Anticoagulantes, varfarina, tromboembolismo venoso.

Os anticoagulantes orais foram descobertos na década de 30 através da observação de vacas que se alimentavam de forragem de trevo-doce (Melilotus alba e $M$. officinalis) deteriorado e apresentavam distúrbios hemorrágicos. Posteriormente, foi demonstrado que esse distúrbio se devia à redução da síntese de protrombina. Link isolou o agente por ele designado de dicumarol e iniciou investigação clínica para a criação de um anticoagulante que mais tarde seria usado como raticida ${ }^{1}$. Link cedeu os direitos da patente do seu composto para a Wisconsin Alumni Research Foundation, donde surgiu o nome "warfarina". No início da década de 50, uma tentativa de suicídio ineficaz de um recruta da Marinha americana

\begin{abstract}
Oral anticoagulants acting via vitamin $\mathrm{K}$ antagonists have long been employed in the clinical practice. However, difficulties related to the management of treatment regimens and complications still persist. Among the complications, bleeding disorders are widely known, but others should also be taken into consideration, such as warfarin-induced skin necrosis. The pathophysiology of this rare but severe complication is still obscure, and its causes remain to be defined. Among possible causes, protein $\mathrm{C}$ and protein $\mathrm{S}$ deficiency, hypersensitivity reactions and VII factor deficiency are the most probable ones. There is an increased incidence of warfarin-induced skin necrosis among middle-aged women, usually affecting breasts and buttocks. The most important treatment measures are immediate discontinuation of the drug, use of unfractionated or lowmolecular-weight heparin at therapeutic doses, use of vitamin $\mathrm{K}$ and, eventually, infusion of fresh-frozen plasma or recombinant activated protein $\mathrm{C}$.
\end{abstract}

Keywords: Anticoagulants, warfarin, venous thromboembolism.

com o raticida levou a um novo estudo clínico em favor da varfarina em 1955; no mesmo ano, o então presidente dos EUA, Eisenhower foi tratado com varfarina depois de um infarto do miocárdio ${ }^{2}$.

A varfarina é um derivado cumarínico, assim como o fenprocumon e o acenocumarol. Sua atividade reside no núcleo 4-hidroxicumarina de uma mistura racêmica de dois isômeros óticos, na qual o enantiômero $\mathrm{S}$ tem ação anticoagulante mais potente que o enantiômero $\mathrm{R}^{3}$. Dentre os cumarínicos, a varfarina é mais utilizada por suas propriedades farmacológicas favoráveis, como início e duração de ação com boa biodisponibilidade, e pela fácil reversão ${ }^{4}$.

1. Doutor. Docente, Universidade Estadual de Londrina (UEL), Londrina, PR.

2. Médico, Serviço de Cirurgia Vascular, Hospital Universitário Regional do Norte do Paraná (HURNP), UEL, Londrina, PR.

3. Acadêmico de Medicina, UEL, Londrina, PR.

Trabalho realizado no Departamento de Clínica Cirúrgica, Serviço de Cirurgia Vascular, Hospital Universitário Regional do Norte do Paraná (HURNP), Universidade Estadual de Londrina (UEL), Londrina, PR.

Não foram declarados conflitos de interesse associados à publicação deste artigo.

Artigo submetido em 04.03.08, aceito em 11.06.09.

J Vasc Bras. 2009;8(4):343-348.

Copyright $@ 2009$ by Sociedade Brasileira de Angiologia e de Cirurgia Vascular 
A ação da varfarina sobre o ciclo da vitamina K ocorre na inibição das enzimas redutases, particularmente a vitamina $\mathrm{K}$ epóxido-redutase, que transformam a vitamina $\mathrm{K}$ em sua forma ativa, vitamina KH2. Como consequência da ação da varfarina ocorre depleção dos fatores de coagulação dependentes da vitamina $\mathrm{K}$ - fatores II, VII, IX, X. Concomitantemente, esses anticoagulantes orais limitam também a produção das proteínas contrarreguladoras da coagulação, as proteínas $\mathrm{C} \mathrm{e} \mathrm{S}^{5}$.

A varfarina é um ácido fraco que apresenta elevada biodisponibilidade, com absorção gastrintestinal vigorosa (em torno de 80 a 100\%), tendo a albumina como principal proteína carreadora. O seu pico de concentração plasmática é atingido em cerca de 2 horas e a sua meia-vida varia de 36 a 42 horas. Acumula-se no fígado, onde interfere diretamente na síntese dos fatores da coagulação e sofre metabolização. A excreção é principalmente feita por via renal, sendo que a idade é o principal determinante do clearance, com declínio de $1 \%$ ao ano, entre as idades de 20 a 70 anos ${ }^{6}$.

O efeito terapêutico das antivitaminas K (AVK) varia de indivíduo para indivíduo, dependendo de fatores genéticos e ambientais que influenciam sua absorção farmacocinética e farmacodinâmica ${ }^{7}$. Os fatores genéticos incluem mutações no gene da enzima do citocromo P450 2C9, que podem reduzir as necessidades de varfarina em seus portadores $^{8,9}$, e a mutação do gene do fator IX, que pode aumentar o risco de sangramento quando do uso das $\mathrm{AVK}^{9-11}$. Há, ainda, uma resistência de origem genética à varfarina que pode aumentar a necessidade de suas doses em até 20 vezes para que se alcance o efeito anticoagulante desejado ${ }^{12}$.

Muitas medicações interagem com a varfarina potencializando ou reduzindo seu efeito ${ }^{1,10,13}$, sendo que a atividade anticoagulante também sofre a influência do tipo de dieta utilizada pelo paciente (mais ou menos rica em vitamina $\mathrm{K})^{14}$. Esses fatos obrigam a um controle terapêutico rigoroso, com correção da dose do anticoagulante de acordo com o tempo de protrombina, que deve ser realizado com a utilização de tromboplastinas com índice de sensibilidade próximo ao do padrão internacional e expresso pela razão normatizada internacional (RNI) ${ }^{15}$.

A varfarina é amplamente usada na prevenção da formação e propagação de trombos, aplicando-se ao tratamento da trombose venosa profunda, embolia pulmonar ${ }^{7,9}$, arritmias cardíacas, valvulopatias, valvuloplastias mecâni- $\operatorname{cas}^{7,10}$, miocardiopatias dilatadas e na cardioversão farmacológica ou elétrica ${ }^{5}$. As suas contraindicações são várias: discrasias sanguíneas associadas a hemorragia ou trombocitopenia, aneurismas cerebrais e dissecções, hemorragia cerebral comprovada ou suspeitada, hipertensão arterial não controlada, ulcerações e lesões ativas do trato gastrointestinal e urinário, cirurgias neurológicas, oftalmológicas e urológicas recentes, traumas recentes, alcoolismo crônico e insuficiência hepática. A idade por si não constitui contraindicação; no entanto, deve-se dar especial atenção à dose estipulada e ao controle terapêutico, já que no idoso, como comentado anteriormente no que tange à excreção renal, a dose prevista é menor para uma anticoagulação eficaz. O risco de queda acidental, acidente vascular cerebral hemorrágico e outras complicações são também potencialmente maiores nesse grupo de pacientes ${ }^{12}$.

As complicações mais importantes e frequentes que podem ocorrer com o uso das AVK são as hemorragias ${ }^{1,10,16,17}$, que podem ter relação com o valor da $\mathrm{RNI}^{18}$. Estudos prospectivos que avaliaram essas complicações mostraram frequência de sangramentos considerados maiores que variaram de 0,4 a $12 \%{ }^{17}$. No entanto, eventos tromboembólicos paroxísticos têm sido reportados desde o início de sua história, incluindo a síndrome do dedo azul, púrpura fulminante e priapismo ${ }^{14}$.

Outras reações adversas incluem reações de hipersensibilidade, icterícia colestática, hepatite, vasculites, náuseas e vômitos, diarreia, alopecia, etc. ${ }^{1,710}$. Também descrito como complicação é o desenvolvimento de uma nova trombose na vigência do tratamento, o que pode ocorrer nos casos de tromboembolismo venoso com uma frequência de 3 a $15 \%{ }^{19}$.

Com menor frequência pode ocorrer a necrose de pele e tecido celular subcutâneo, que está presente em cerca de 0,01 a $0,1 \%$ dos $\operatorname{casos}^{13,17,20-24}$. A primeira descrição de necrose cutânea induzida por varfarina (NCIV) foi feita por Flood et al. ${ }^{15}$. Na ocasião, porém, foi erroneamente diagnosticada como tromboflebite migratória. Somente no final da década de 50 é que se estabeleceu a relação de causa entre a necrose tecidual e o uso de anticoagulantes orais $^{15,18}$. Segundo Chan et al. ${ }^{16}$, foi Verhagen, em 1954, quem estabeleceu essa relação quando descreveu 13 casos. Cabe lembrar que esta complicação pode ocorrer com qualquer anticoagulante oral que tenha o antagonismo à vitamina K como mecanismo de ação. 
De etiologia desconhecida, ainda é difícil determinar qual paciente poderá vir a desenvolver esse efeito colateral. Trombose, hipersensibilidade, hemorragia, deficiência de fator VII, deficiência de proteína $\mathrm{C}$ e toxicidade por varfarina têm sidos sugeridos como possíveis causas desencadeantes dessa complicação ${ }^{25}$. Ela pode ser observada também em pacientes portadores de lupus eritematoso sistêmico, assim como em associação a deficiências de proteínas $\mathrm{C}$ e/ou $\mathrm{S}$, anticoagulante lúpico e insuficiência renal. Em outras situações, tem sido observada a ocorrência de lesões em pacientes recebendo tratamento para trombose venosa profunda e tromboembolismo pulmonar com doses diárias de varfarina superiores a $10 \mathrm{mg}$, que são consideradas doses iniciais elevadas, o que pode sugerir o não emprego dessas doses na fase inicial de tratamento ${ }^{17,25}$.

As proteínas anticoagulantes naturais $\mathrm{C}$ e $\mathrm{S}$ e o fator VII, de meia-vida curta, têm queda mais rápida de suas concentrações do que os outros fatores envolvidos na prócoagulação (fatores II, IX e X), de meia-vida entre 20 e 60 horas. Como consequência, a redução não contrabalanceada dessas proteínas induz a um período de potencial efeito pró-trombótico no qual podem surgir as lesões necróticas entre o terceiro e sexto dia de uso do anticoagulante oral em $90 \%$ dos casos ${ }^{18,20}$. Isto leva à recomendação do uso concomitante de heparina nos primeiros dias de tratamento com as $\mathrm{AVK}^{5}$.

A patogênese da NCIV ainda é considerada incerta. Acredita-se que ocorra uma falha em um ou mais fatores anticoagulantes naturais na microvasculatura junto à queda farmacologicamente induzida dos fatores de coagulação dependentes da vitamina $\mathrm{K}$.

O conhecimento a respeito das funções das proteínas C, S e antitrombina III tem ajudado na melhor elucidação dos mecanismos envolvidos nessa complicação. Foi constatada deficiência hereditária de proteína $\mathrm{C}$ em um terço dos pacientes que desenvolveram $\mathrm{NCIV}^{14,16,21}$. Este dado confere com a frequência, estimada em 1:350 ${ }^{19}$, com que pacientes assintomáticos com defíciência na proteína $\mathrm{C}$ apresentam o evento necrocutâneo. Entretanto, não existe apenas este risco; outras hipercoagulopatias podem estar envolvidas, como a deficiência de proteína $\mathrm{S}^{6,26}$, de antitrombina $\mathrm{III}^{27}$, fator $\mathrm{V}$ de Leiden ${ }^{28}$ e anticoagulante lúpi$\mathrm{co}^{29}$.

A NCIV é anunciada por parestesia ou sensação de pressão associada a lesões eritematosas de surgimento sú- bito com bordas poucos demarcadas e geralmente dolorosas, que evoluem com pontos hemorrágicos. O edema do tecido dérmico e subcutâneo produz lesão com aparência de casca de laranja. Após 24 horas de evolução, petéquias e bolhas hemorrágicas envolvem a área. $\mathrm{O}$ aparecimento destas bolhas hemorrágicas evidencia um estágio irreversível da necrose de pele. Eventualmente podem surgir úlceras.

Esta condição desenvolve-se majoritariamente em mulheres de meia-idade que estão em menopausa, obesas e em tratamento de trombose venosa profunda ou tromboembolismo pulmonar ${ }^{30}$, sendo infrequente nos pacientes com insuficiência cerebrovascular e fibrilação atrial ${ }^{16}$. A razão para esta predominância no sexo feminino não está esclarecida.

Os primeiros sintomas aparecem entre o primeiro e $10^{\circ}$ dia de tratamento com varfarina, com as lesões geralmente surgindo entre o terceiro e sexto dia de uso. Existem alguns relatos de quadros de NCIV iniciados tardiamente, após 16 dias de tratamento ${ }^{31}$. Mostra-se pouco provável que esses casos tenham como causa a terapia anticoagulante; mesmo assim, como os níveis do anticoagulante permanecem flutuantes, o diagnóstico etiológico de NCIV deve ser considerado ${ }^{32}$.

Os locais com maior abundância de tecido adiposo são os mais frequentemente atingidos. Na mulher, a área mais acometida é a das mamas seguida pelas nádegas e coxas; ocasionalmente, o tronco, a face e as extremidades são envolvidas. Já no homem, é difícil ocorrer necrose cutânea no tronco; no entanto, é mais comum no pênis, apesar de também ser rara esta apresentação ${ }^{33}$.

O diagnóstico é essencialmente clínico. Podem ser realizados exames histopatológicos das lesões para melhor comprová-las; no entanto, a história clínica geralmente é soberana. O diagnóstico diferencial de NCIV deve ser feito com fasciíte necrosante, gangrena venosa e outras causas de necrose de pele, sendo que a entidade também pode ser confundida com outras doenças dermatológicas ${ }^{16}$. A biópsia pode mostrar, inicialmente, injúria na microvasculatura pós-capilar com depósitos de fibrina nas vênulas e pequenas veias com hemorragia poupando as arteríolas e em um estágio mais tardio apresenta necrose difusa na derme e tecido subcutâneo e trombose microvascular ${ }^{25}$. A imunoflorescência direta não mostra alterações específicas, sendo descritos casos de depósito de IgM e/ou de C3, 
possivelmente pela presença concomitante de mononucleose infecciosa ${ }^{34}$. Alguns autores postularam que a varfarina possui efeito tóxico sobre as junções pré-capilar e arteriolar da derme. De acordo com essa teoria, a varfarina provocaria lesão endotelial levando à liberação de citocinas pró-inflamatórias, dilatação e ruptura dos capilares, resultando em petéquias, equimoses e lesões necróticas $^{30,35,36}$. No entanto, não foi demonstrado um mecanismo imunológico associado, como é observado na lesão por heparina $^{37}$.

Não existe um único tratamento específico indicado por especialista para a NCIV e muito menos estudos randomizados e controlados. O tratamento da fase inicial é prevenir a evolução do quadro para necrose cutânea com a suspensão imediata do anticoagulante oral e emprego de vitamina $\mathrm{K}, 10-20 \mathrm{mg}$, ambos por via endovenosa. Pode-se utilizar também a infusão de plasma fresco congelado na dose de $10-20 \mathrm{~mL} / \mathrm{kg}^{20}$. Estas medidas visam repor as proteínas $\mathrm{C}, \mathrm{S}$ e fatores de coagulação, assim como estimular a produção (vitamina $\mathrm{K}$ ). Na deficiência de proteína $\mathrm{C}$, deve-se repor com concentrado de anticorpo monoclonal purificado $^{24}$. No entanto, esta terapêutica não pode ser recomendada por tempo prolongado em decorrência de seu alto custo.

A interrupção do tratamento com varfarina não cura ou altera a progressão da enfermidade. Portanto, deve-se manter anticoagulação com heparina ${ }^{30}$. Apesar de o uso de heparina também estar associado a necrose cutânea, não existem relatos na literatura de progressão dessa complicação com o uso de heparina depois da indução dela pela varfarina. O mecanismo é diferente: a heparina pode induzir a trombocitopenia de natureza imune e causar hemorragia ou ainda levar a uma complicação clínica mais importante, que é o desenvolvimento paradoxal de trombose ${ }^{34}$.

Mesmo com as medidas descritas anteriormente, a maioria dos casos necessita de debridamentos ou até amputação $^{16}$. A prostaciclina tem sido utilizada com resultados clínicos e histológicos promissores; no entanto, a validação do seu uso carece de ensaios clínicos randomizados e controlados ${ }^{30,38}$. É um potente vasodilatador e inibidor da antiagregação plaquetária, sendo utilizado em desordens vasculares e trombóticas.

Assim que o quadro tenha se estabilizado, é reinstituída a terapia com varfarina. No entanto, a reintrodução deve ser gradual e em baixas doses ${ }^{8,39}$. A heparina nesta fase deve ser mantida por ao menos 1 semana. É essencial para a continuidade da terapêutica saber qual é a causa base, devendo-se, desta maneira, fazer a dosagem de proteína $\mathrm{C}, \mathrm{S}$, fibrinogênio e produtos da degradação da fibrina. Estas dosagens não podem ser feitas na vigência do tratamento com AVK. É preferível investigar pessoas suspeitas de terem deficiência dos fatores de coagulação dependentes da vitamina $\mathrm{K}$ depois da anticoagulação oral ter sido descontinuada por pelo menos uma semana, devendo ser estudados durante o tratamento com heparina, que não altera os níveis plasmáticos da proteína $\mathrm{C}$.

A NCIV é uma situação pouco habitual, porém de evolução grave com possibilidade de amputação e morte. Sendo assim, a detecção precoce e a atenção ao grupo de maior risco é essencial para uma rápida instituição do tratamento. Tem particular importância o diagnóstico diferencial com gangrena venosa resultante da trombose venosa profunda e que tem a anticoagulação como indicação de tratamento. O conhecimento adquirido através dos exames para pesquisa de trombofilia nos casos de pacientes com histórico familiar para tromboembolismo venoso é de grande valia nos cuidados com a terapia antitrombótica instituída e na prevenção dessa complicação.

\section{Referências}

1. Link KP. The discovery of dicoumarol and its sequels. Circulation. 1959;19:97-107.

2. Scully, M. Warfarin therapy: rat poison and the prevention of thrombosis. Bioquemist. 2002;27:15-7.

3. O'Reilly RA. Vitamin K and the oral anticoagulant drugs. Annu Rev Med. 1976;27:245-61.

4. Sardinha WE, Silvestre JM. Anticoagulantes e fibrinolíticos. In: Thomaz JB, Belczak CE, editores. Tratado de flebologia e linfologia. Rio de Janeiro: Rubio; 2006. p. 511-32.

5. Batlouni M. Anticoagulantes orais. In: Batlouni M, Ramires JA, editores. Farmacologia e terapêutica cardiovascular. $2^{\mathrm{a}}$ ed. São Paulo: Atheneu; 2004. p. 351-66.

6. Silveira PR, Panico MD. Anticoagulantes. In: Brito CJ, Duque A, Merlo I, Murilo R, Fonseca VL, editores. Cirurgia vascular. Rio de Janeiro: Revinter; 2002. p. 375-89.

7. Santos FC, Maffei FH, de Carvalho LR, et al. Complicações da terapia anticoagulante com warfarina em pacientes com doença vascular periférica: estudo de coorte prospectivo. J Vasc Bras. 2006;5:194-202.

8. Ansell J, Hirsh J, Hylek E, et al. Pharmacology and management of the vitamin $\mathrm{K}$ antagonists: American College of Chest Physicians Evidence-Based Clinical Practice Guidelines (8th Edition). Chest. 2008;133:160S-98S.

9. Schulman S. Clinical practice. Care of patients receiving long-term anticoagulant therapy. $\mathrm{N}$ Engl J Med. 2003;349:675-83. 
10. Cortelazzo S, Finazzi G, Viero P, et al. Thrombotic and hemorrhagic complications in patients with mechanical heart valve prosthesis attending an anticoagulation clinic. Thromb Haemost. 1993;69:316-20.

11. Maffei FH, Rollo HA. Trombose venosa profunda dos membros inferiores: incidência, patologia, fisiopatologia e diagnóstico. In: Maffei FH, Lastória S, Yoshida WB, Rollo HA. Doenças vasculares periféricas. $3^{\mathrm{a}}$ ed. São Paulo: Medsi; 2002. p. 1363-86.

12. Gersh BJ, Opie LH. Antitrrombotic agents: platelet inhibitors, anticoagulants, and fibrinolysis. In: Opie LH, editor. Drugs for the heart. 4th ed. Philadelphia: WB Saunders; 1995. p. 248-87.

13. Schulman S, Beyth R, Kearon C, Levine MN; American College of Chest Physicians. Hemorrhagic complication of anticoagulant and thrombolytic treatment: American College of Chest Physicians Evidence-Based Clinical Practice Guidelines (8th Edition). Chest. 2008;133:257S-98S.

14. Sallah S, Thomas DP, Roberts HR. Warfarin and heparin-induced skin necrosis and the purple toe syndrome: infrequent complications of anticoagulant treatment. Thromb Haemost. 1997;78:785-90.

15. Flood EP, Redish MH, Bociek SJ, Shapiro S. Thrombophlebitis migrans disseminate: report of a case in which gangrene of the breast occurred. Observations on the therapeutic use of dicumarol (3,3'methylenebis(4-hydroxycoumarin)). N Y State J Med. 1943;43:1121-4.

16. Chan YC, Valenti D, Mansfield AO, Stansby G. Warfarin induced skin necrosis. Br J Surg. 2000;87;266-72.

17. Eby CS. Warfarin-induced skin necrosis. Hematol Oncol Clin North Am. 1993;7:1291-300.

18. Lewandowski K, Zawilska K. Protein C concentrate in the treatment of warfarin-induced skin necrosis in the protein $\mathrm{C}$ deficiency. Thromb Haemost. 1994;71:395.

19. Lane DA, Mannucci PM, Bauer KA, et al. Inherited thrombophilia: Part 1. Thromb Haemost. 1996;76:651-62.

20. Essex DW, Wynn SS, Jin DK. Late-onset warfarin-induced skin necrosis: case report and review of the literature. Am J Hematol. 1998;57:233-7.

21. Gladson CL, Groncy P, Griffin JH. Coumarin necrosis, neonatal purpura fulminans, and protein $\mathrm{C}$ deficiency. Arch Dermatol. 1987;123:1701a-6a.

22. Ad-El DD, Meirovitz A, Weinberg A, et al. Warfarin skin necrosis: local and systemic factors. $\mathrm{Br} \mathrm{J}$ Plast Surg. 2000;53:624-6.

23. Warkentin TE, Sikov WM, Lillicrap DP. Multicentric warfarin-induced skin necrosis complicating heparin-induced thrombocytopenia. Am J Hematol. 1999;62:44-8.

24. Harenberg J, Hoffmann U, Huhle G, Winkler M, Bayerl C. Cutaneous reactions to anticoagulants. Recognition and management. Am J Clin Dermatol. 2001;2:69-75.

25. Cole MS, Minifee PK, Wolma FJ. Coumarin necrosis: a review of the literature. Surgery. 1988;103:271-7.

26. Grimaudo V, Gueissaz F, Hauert J, Sarraj A, Kruithof EK, Bachmann F. Necrosis of skin induced by coumarin in a patient deficient in protein S. BMJ. 1989;298:233-4.

27. Kiehl R, Hellstern P, Wenzel E. Hereditary antithrombin III (AT III) deficiency and atypical localization of a coumarin necrosis. Thromb Res. 1987;45:191-3.
28. Dahlbäck B. New molecular insights into the genetics of thrombophilia. Resistance to activated protein $\mathrm{C}$ caused by Arg506 to Gln mutation in factor $\mathrm{V}$ as a pathogenic risk factor for venous thrombosis. Thromb Haemost. 1995;74:139-48.

29. Moreb J, Kitchens CS. Acquired functional protein S deficiency, cerebral venous thrombosis, and coumarin skin necrosis in association with antiphospholipid syndrome: report of two cases. Am J Med. 1989;87:207-10.

30. Nalbandian RM, Malder IJ, Barrett JF, Pearce JF, Rupp EC. Petechiae, ecchymoses, and necrosis of skin induced by coumarin congeners. Rare, occasionally lethal complication of anticoagulant therapy. JAMA. 1965;192:603-8.

31. Goldberg SL, Orthner CL, Yalisove BL, Elgart ML, Kessler CM. Skin necrosis following prolonged administration coumarin in a patient with inherited protein S deficiency. Am J Hematol. 1991;38:64-6.

32. Kagan RJ, Glassford GH. Coumadin-induced breast necrosis. Am Surg. 1981;47:509-10.

33. Barkley C, Badakament RA, Metz EM, Nesbitt J, Drago JR. Coumarin necrosis of the penis. J Urol. 1989;141:946-8.

34. Hermes B, Haas N, Henz BM. Immunopathological events of adverse cutaneous reactions to coumarin and heparin. Acta Derm Venereol. 1997;77:35-8.

35. Franson TR, Rose HD, Spivey MR, Maroney J, Libnoch JA. Late-onset, warfarin-caused necrosis occurring in a patient with infectious mononucleosis. Arch Dermatol. 1984;120:927-31.

36. Schleicher SM, Fricker MP. Coumadin necrosis. Arch Dermatol. 1980;116:444-5.

37. Shahak A, Pósán E, Szücs G, Rigó J, Boda Z. Coumarininduced skin necrosis following heparin-induced thrombocytopenia and thrombosis: a case report. Angiology. 1996;47:725-7.

38. Srinivasan AF, Rice L, Bartholomew JR, et al. Warfarininduced skin necrosis and venous limb gangrene in the setting of heparin-induced thrombocytopenia. Arch Intern Med. 2004; $164: 66-70$.

39. Norris PG. Warfarin skin necrosis treated with prostacyclin. Clin Exp Dermatol. 1987;12:370-2.

Correspondência:

José Manoel da Silva Silvestre

Hospital Universitário

Rua Robert Koch, $\mathrm{s} / \mathrm{n}^{\circ}$, Bairro Cervejaria

CEP 86010-020 - Londrina, PR

Tel.: (43) 3371.2645/2000, (43) 3339.6347,

(43) 9995.8284

E-mail: silvestrejms@sercomtel.com.br

\section{Contribuições dos autores:}

Concepção e desenho do estudo: JMSS, ISP, FT

Análise e interpretação dos dados: ISP, FT

Coleta de dados: ISP, FT

Redação do artigo: ISP, FT, JMSS

Revisão crítica do texto: JMSS, WES, DMF 
348 J Vasc Bras 2009, Vol. 8, $N^{\circ} 4$

Aprovação final do artigo*: JMSS, FT, WES, ISP, DMF Análise estatística: N/A

Responsabilidade geral pelo estudo: JMSS
Necrose cutânea induzida por antagonistas da vitamina K - Silvestre JM et al.

Informações sobre financiamento: N/A

*Todos os autores leram e aprovaram a versão final submetida ao J Vasc Bras. 